\title{
The Reaction Extraction Combining Crystallization for Growth of Sodium Chloride in a Spray Fluidized Bed Crystallizer
}

\author{
Dan Zheng $\mathbb{D}^{1}{ }^{1}$ Jie Yan, ${ }^{1}$ Jun Chen, ${ }^{2}$ and Zeqin Wang ${ }^{2}$ \\ ${ }^{1}$ Department of Chemical Engineering, Sichuan University of Science \& Engineering, Zigong, Sichuan 643000, China \\ ${ }^{2}$ Sichuan Key Laboratory of Rock and Well Salt, Light Industry Design and Research Institute, Zigong, Sichuan 643000, China \\ Correspondence should be addressed to Dan Zheng; zhengdan0830@163.com
}

Received 6 August 2020; Accepted 10 November 2020; Published 29 November 2020

Academic Editor: Cláudia G. Silva

Copyright (c) 2020 Dan Zheng et al. This is an open access article distributed under the Creative Commons Attribution License, which permits unrestricted use, distribution, and reproduction in any medium, provided the original work is properly cited.

At present, the crystal size of sodium chloride prepared by a traditional crystallization process (such as stirred crystallization) is inhomogeneous, and it has a great quantity of fine grains in crystallizer. This work presents a novel approach for the growth of sodium chloride from supersaturated solutions by reaction-extractive crystallization in a spray fluidized bed crystallizer (SFBC), in which sodium sulfate solution is transformed into potassium chloride and sulphuric acid based on a reactive extractioncrystallization process using triisooctylamine (TOL) in $n$-octanol as the extraction system. This paper mainly studies the effect of operating conditions (e.g., circulation flow rate, velocity ratio of oil and aqueous phases, crystallization temperature, hydraulic residence time, and feed velocity) on the crystal size distribution (CSD) during the crystallization process of sodium chloride in a SFBC. Experimental results show that the optimum conditions are $1.0362 \mathrm{~m} / \mathrm{s}$ as the best circulation flow rate, $9.5: 8.5$ as the best velocity ratio of oil and aqueous phases, $313 \mathrm{~K}$ as the best temperature, $4320 \mathrm{~s}$ as residence time, and $8 \mathrm{~mL}^{-\mathrm{min}^{-1}}$ as the best feed velocity. Meanwhile, the proposed extraction kinetic model about extraction rates is developed and validated against data from the SFBC. And it proves that the reactive extraction system is controlled by diffusion and chemical reaction. Analysis of the extraction kinetic model and comparison with experiments reveal that the extraction kinetic model results are in well agreement with experiments. Furthermore, the uniform and large crystals can be obtained in a spray fluidized bed crystallizer without special concentration since extraction and crystallization are carried out in the same equipment. In addition, all of the sodium chloride products prepared under the optimal conditions in SFBC show a better CSD performance than the stirred crystallization. This research demonstrates that this process enables controlling the crystal size in a rather wide range, thus further underlining the potential of this technique for applications in the crystallization industry.

\section{Introduction}

As a chemical raw material, well salt is widely used in the agricultural, chemical, biochemical, and food industries [1]. The development of salt production contributed to the prosperity of border economy during the ancient times [2]. However, the rapid development of sea salt and lake salt makes the development of well salt difficult. The reasons are as follows: (1) the sea salt has the wonderful quality (e.g., the average content of sodium chloride in sea salt is $91.24 \%$, the average whiteness is $58.57 \%$, and the average particle size is $91.41 \%$ ) [3]. Therefore, it is an ideal manufactured material for chemical salt and edible salt. (2) The average content of sodium chloride in lake salt is high, and the production cost of lake salt is lower than that of well salt in industry [4]. Hence, economical and efficient crystallization technologies are desired for industrial production of sodium chloride in the past few years [5]. However, the entire production process of well salt is complicated and the investment is high due to the lack of large-scale salt making facility and related technical research [6]. Consequently, improving the purity of sodium chloride in well salts and reducing production costs are important means to promote the development of the salt industry [7].

The reaction extraction is prospective in sodium chloride crystallization with the advantages of easy scale-up and low energy consumption [8]. Crystallization by reactive extraction is considered a promising approach for the 
purification of sodium chloride, which is generally acknowledged by academic circle [9]. In the reaction extraction combining crystallization, the mixed solution of hydrochloric acid and sodium sulfate contacts with the extractant, triisooctylamine (TOL) in $n$-octanol, and the following reaction takes place:

$$
\mathrm{Na}_{2} \mathrm{SO}_{4}+\mathrm{HCI} \longrightarrow \mathrm{NaCI}+\mathrm{H}_{2} \mathrm{SO}_{4} \text {. }
$$

In general, it can be seen from Equation (1) that this reaction does not occur without external stimuli. In the reaction extraction process, as sulphuric acid is extracted from the aqueous phase by an extractant, the equilibrium shifts to the right, yielding sodium chloride in the aqueous phase. Moreover, the extractant could be recycled and reused. This novel coupled process is first suggested by Kosswig and Praun in the soda industry. They put forward a new process to produce soda using organic amines which could be reused through distillation instead of inorganic ammonia [10].

With the continuous improvement in the requirements for crystal quality and environmental protection in global industry, currently, it has become a prime target of reactive extraction-crystallization to improve the yield and quality of crystal at the same time [11]. Consequently, the design for crystallizer strives to achieve safety and reliability, advanced technology, and economic rationality while meeting production requirements [12]. At present, as shown in Figure 1, over $90 \%$ of sodium chloride production is from the stirred crystallization [13]. As far as we know, although the operation is convenient, the crystals are very easy to collide with the impeller during the growth process in a stirred crystallizer and ultimately affect the main size of the crystal products [14].

The process of fluidized crystallization comprises the characteristics of variable crystal movements and complex fluidization behavior [15]. Hence, it has received more attention due to its high mass transferring efficiency and well multiphase mixing compared to a stirred crystallizer [16]. Hence, a reactive extraction-crystallization coupled process needs to be investigated systematically in a SFBC. Figure 2 shows the flowchart of the system including extractive crystallization in SFBC. The entire reaction system mainly includes feeding system, fluidized system, and sampling system. This process is controlled by continuous circulation operation, and it is only required to clean the pump and pipeline regularly. On the contrary, the recycling of the extractant will greatly reduce production costs but will also reduce waste liquid discharge as a result of environmental pollution [17]. However, the crystallization of sodium chloride by the coupled process in SFBC has rarely been studied and documented, and the extraction mechanism is investigated not combining with the coupled process but just in the conventional way in the previous reports.

In this study, we present a reaction extraction combining crystallization technique in a SFBC for the growth of sodium chloride from supersaturated solution. The experiment operating the SFBC in a continuous mode is performed to explore the effects of the important parameters (velocity ratio of oil and aqueous phases, crystallization temperature, hydraulic residence time, and feed velocity) on the desired crystal size distribution, and the optimal conditions are determined. The coupled process is expected to offer a reference for the industrial application. This study provides a new perspective to the understanding of the crystallization of sodium chloride by the coupled process in industry in the recent years.

\section{Experimental Methods}

2.1. Chemicals. Triisooctylamine (TOL) (mass fraction $\geq 95 \%$ ) and $n$-octanol are provided by Langfang Qianyao Technology co., Ltd., China. Sodium sulfate (AR) and sodium hydroxide (AR) are purchased from Henan Mingzhixin Chemical Products co., Ltd., China. And hydrochloric acid (AR, mass fraction 36-38\%) is from Pengcai Chemical Works, China. The aqueous solutions are prepared using deionized water. The supersaturated solution of sodium chloride is prepared from the deionized water. The tiny crystals of sodium chloride are placed inside the SFBC as the crystal seed material. All chemicals are used without further purification.

\subsection{Experiment}

2.2.1. Experimental Setup and Procedure. The mixed solutions of hydrochloric acid and sodium sulfate with different experimental conditions and the extractant of the triisooctylamine (TOL) in $n$-octanol are prepared, respectively. The thermostatic water bath is controlled at temperatures of $308,311,313,318$, and $321 \mathrm{~K}$ with a temperature uncertainty of $\pm 0.5 \mathrm{~K}$.

A schematic diagram of the fluidized crystallization is shown in Figure 3(a). This study used a recirculation mode, and all experiments are conducted at room temperature. The oil and aqueous phases are both delivered by the recirculation pumps and preheated in a thermostatic water bath before entering the spray fluidized bed crystallizer. The geometry of the SFBC applied in this work is illustrated in Figure 3(b). The SFBC that has V type wall has the fast intermediate velocity and slow peripheral velocity [12]. The whole bed is $0.29 \mathrm{~m}$ in height, and the bottom part as a spray zone is made up of a $0.04 \mathrm{~m}$ cylinder with $0.01 \mathrm{~m}$ inlet diameter. The installation height of the fluidization zone is $0.09 \mathrm{~m}$ above the bottom, and the diameter is $0.03 \mathrm{~m}$. The recirculation pump is turned on to suspend the crystal seeds to a desired bed expansion level and homogenize the solution [18]. The two phases in the mixed solution are mixed vigorously after flowing out of the top of the crystallizer. A buffer tank between crystallizer and phase-separation tank guarantees that the phases in the mixed solution can be separated rapidly, and it can reduce the residence time. In order to increase the axial velocity of the fluid and avoid the crystal suspension in the solution, a guide tube is added into the phase-separation tank and some holes are left on it. In addition, the uneven diameter of the crystallizer inlet is to promote the mixing degree of the oil and aqueous phases in the spray zone of crystallizer. At the end of experiment, the 


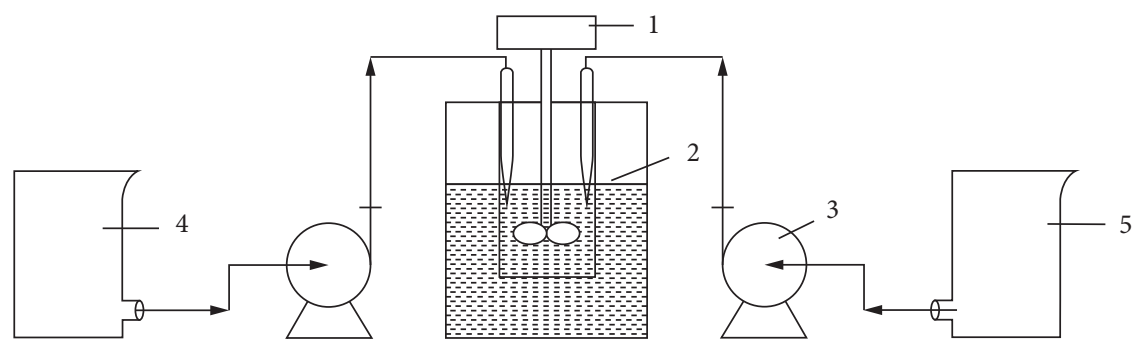

FiguRE 1: Experimental setup for stirred crystallization. 1, agitator; 2, crystallizer; 3, peristaltic pump; 4, mixed solution (aqueous phase); 5, extractant (oil phase).

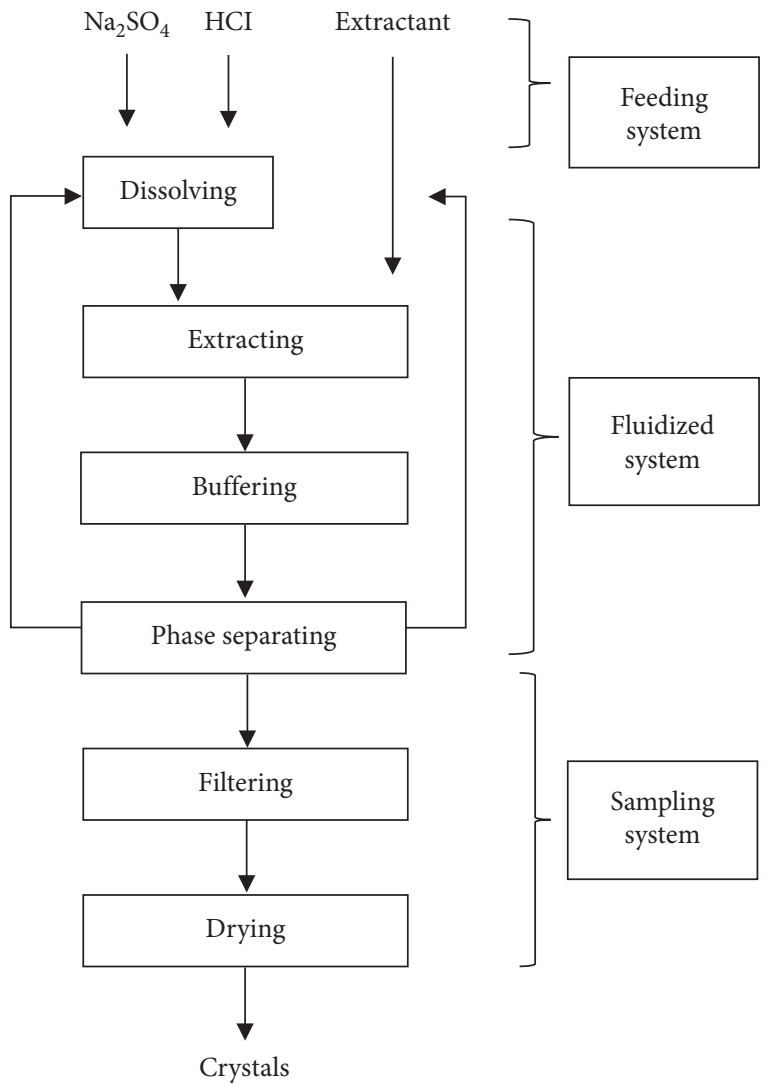

FIGURE 2: The process steps for preparing pure sodium chloride in SFBC.

generated crystals accumulating at the crystallizer bottom are withdrawn and are oven-dried at $120^{\circ} \mathrm{C}$ for about $1-1.5 \mathrm{~h}$

In this system, the state of extraction reaction changes from "static" to "dynamic" in SFBC, which can effectively prevent crystallizer clogging up. Meanwhile, according to the crystallization kinetics principle, the "dynamic" state of the extraction reaction can promote crystallization, and it is also conducive to the floating of the oil phase with low density and overflow from the top of the crystallizer. Each experiment is repeated to assure reproducibility of the results. A summary for the conditions applied during experiment campaigns is given in Table 1.
2.2.2. Extraction Kinetics Model. We assume that the extraction of sulphuric acid by TOL could be described as follows, respectively:

$$
\begin{aligned}
\mathrm{H}_{2} \mathrm{SO}_{4(a)} & \rightleftharpoons \mathrm{H}_{2} \mathrm{SO}_{4(a o)}, \\
\mathrm{TOL}_{(o)} & \rightleftharpoons \mathrm{TOL}_{(a o)}, \\
\mathrm{H}_{2} \mathrm{SO}_{4(a o)}+\mathrm{TOL}_{(a o)} & \rightleftharpoons H_{2} \mathrm{SO}_{4} \cdot \mathrm{TOL}_{(a o)}, \\
\mathrm{H}_{2} \mathrm{SO}_{4} \cdot \mathrm{TOL}_{(a o)} & \rightleftharpoons \mathrm{H}_{2} \mathrm{SO}_{4} \cdot \mathrm{TOL}_{(o)},
\end{aligned}
$$

where $(a),(o)$, and $(a o)$ represent the aqueous phase, oil phase, and the phase interface of two phases, respectively. The extraction rate $(R)$ can be described as follows:

$$
R=\frac{V}{A} \frac{\mathrm{d}\left[H_{2} \mathrm{SO}_{4} \cdot \mathrm{TOL}_{(a o)}\right]}{\mathrm{d} t}=k_{f}\left[H_{2} \mathrm{SO}_{4}\right]_{(a)}-k_{b}\left[H_{2} \mathrm{SO}_{4}\right]_{(o)} \text {, }
$$

where $k_{f}$ and $k_{b}$ represent forward and backward rate constants. The values of $k_{f}$ and $k_{b}$ can be simply deduced by the integrative of equation (6):

$$
\begin{aligned}
\frac{a_{i}-a_{e}}{a_{i}} \ln \frac{a_{i}-a_{e}}{a_{t}-a_{e}} & =\frac{A}{V} k_{f} t, \\
\frac{a_{e}}{a_{i}} \ln \frac{a_{i}-a_{e}}{a_{t}-a_{e}} & =\frac{A}{V} k_{b} t,
\end{aligned}
$$

where $a_{i}, a_{e}$, and $a_{t}$ represent initial concentration, equilibrium concentration, and concentration of at time $t$ of the sulphuric acid. The experimental extraction rate can be obtained by substituting the values of $k_{b}$ and $k_{f}$ back to equation (6).

Assume that the forward and backward reactions are both first order. Equations (2) and (4) belong to slow reaction. The changes in the concentrations of sulphuric acid in the aqueous phase can be described as follows:

$$
\begin{aligned}
&-\frac{\mathrm{d} C_{\mathrm{H}_{2} \mathrm{SO}_{4(a)}}}{\mathrm{d} t} \rightleftharpoons k\left(C_{\mathrm{H}_{2} \mathrm{SO}_{4(a)}}-C_{\mathrm{H}_{2} \mathrm{SO}_{4(a o)}}\right), \\
& \frac{\mathrm{d} C_{\mathrm{H}_{2} \mathrm{SO}_{4} \cdot \mathrm{TOL}_{(a o)}}^{\mathrm{d} t}}{} \rightleftharpoons k^{+} C_{\mathrm{H}_{2} \mathrm{SO}_{4(a o)}} C_{\mathrm{TOL}_{(a o)}}-k^{-} C_{\mathrm{H}_{2} \mathrm{SO}_{4} \cdot \mathrm{TOL}_{(a o)}}
\end{aligned}
$$




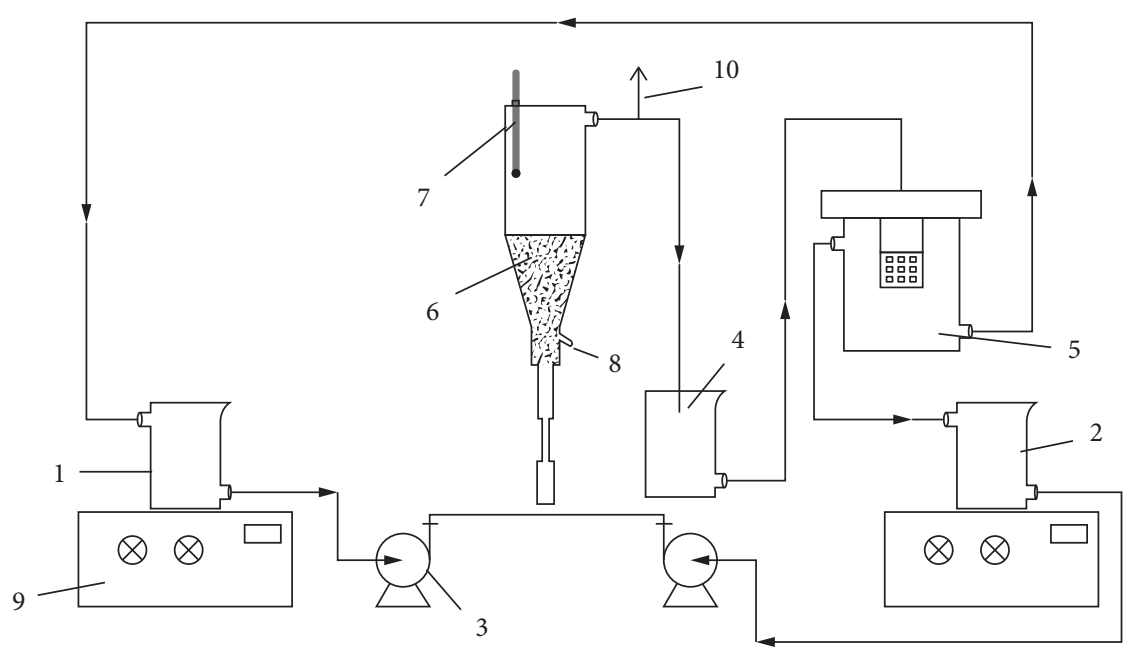

(a)

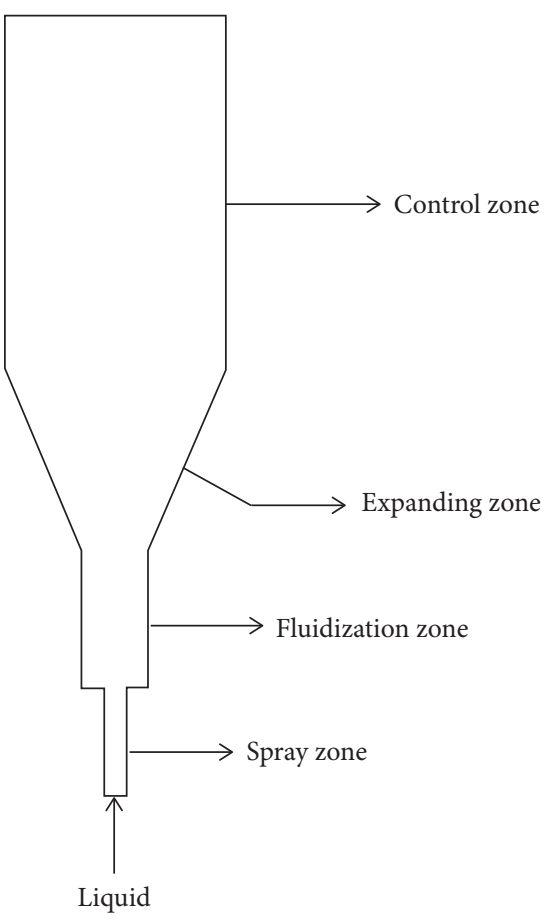

(b)

Figure 3: (a) The schematic diagram of the experimental setup; (b) the structure chart of the spray fluidized bed crystallizer. 1, aqueous phase feed; 2, oil phase feed; 3, recirculation pump; 4, buffer tank; 5, phase-separation tank; 6, crystallizer; 7, temperature probe; 8 , reclaiming port; 9 , thermostatic water bath; 10 , tee.

TABLE 1: Experiment conditions.

\begin{tabular}{|c|c|c|c|c|c|}
\hline Experimental parameters & Condition 1 & Condition 2 & Condition 3 & Condition 4 & Condition 5 \\
\hline Velocity ratio of oil and aqueous phases & $9.5: 8$ & $9.5: 8.2$ & $9.5: 8.5$ & $9.5: 8.6$ & $9.5: 9$ \\
\hline Crystallization temperature $(\mathrm{K})$ & 308 & 311 & 313 & 318 & 321 \\
\hline Hydraulic residence time (s) & 3180 & 3420 & 4320 & 4860 & 5160 \\
\hline Feed velocity $\left(\mathrm{mL} \cdot \mathrm{min}^{-1}\right)$ & 6 & 7 & 8 & 9 & 10 \\
\hline Circulation flow rate $(\mathrm{m} / \mathrm{s})$ & 0.3454 & 0.6908 & 1.0362 & 1.3862 & - \\
\hline
\end{tabular}

Equations (3) and (5) belong to fast reaction. The changes in the concentrations of TOL in the oil phase can be described as follows:

$$
\begin{gathered}
\frac{\mathrm{d} C_{\mathrm{TOL}_{(o)}}}{\mathrm{d} t} \rightleftharpoons \frac{\mathrm{d} C_{\mathrm{TOL}_{(a o)}}}{\mathrm{d} t}, \\
\frac{\mathrm{d}_{\mathrm{H}_{2} \mathrm{SO}_{4} \cdot \mathrm{TOL}_{(a o)}}}{\mathrm{d} t} \rightleftharpoons \frac{\mathrm{d} C_{\mathrm{H}_{2} \mathrm{SO}_{4} \cdot \mathrm{TOL}_{(o)}} .}{\mathrm{d} t} .
\end{gathered}
$$

Substituting equations (10) and (12) into equation (6), the extraction rate for can be approximately evaluated as follows:

$$
R=\frac{k k^{+} C_{H_{2} \mathrm{SO}_{4(a)}} C_{\mathrm{TOL}_{(a)}}-k k^{-} C_{\mathrm{H}_{2} \mathrm{SO}_{4} \cdot \mathrm{TOL}_{(o)}}}{k^{+} C_{\mathrm{TOL}}(o)} \times \frac{V}{A} .
$$

When $\quad t$ approaches $0, \quad C_{H_{2} \mathrm{SO}_{4(a)}} \longrightarrow C_{\mathrm{H}_{2} \mathrm{SO}_{4(a, 0)}}$ $C_{\mathrm{TOL}_{(a o)}} \longrightarrow C_{\mathrm{TOL}_{(a, 0)}} C_{\mathrm{H}_{2} \mathrm{SO}_{4} \cdot \mathrm{TOL}_{(o)}} \longrightarrow 0$, and equation (13) can be simplified as follows:

$$
R=\frac{k k^{+} C_{H_{2} \mathrm{SO}_{4(a, 0)}} C_{\mathrm{TOL}_{(a 0,0)}}}{k^{+} C_{\mathrm{TOL}_{(0,0)}}+k} \times \frac{V}{A}
$$

$K=\mathrm{kV} / A$ and $K^{+}=k^{+} \mathrm{V} / A$ are introduced to simplify equation (14), and we can find this as follows:

$$
R=\frac{C_{H_{2} \mathrm{SO}_{4(a, 0)}}}{\left(1 / K^{+} C_{\mathrm{TOL}_{(0,0)}}^{+}\right)+(1 / K)},
$$

where $K, K^{+}$is the constant of extraction rate. This extraction kinetic model involves the initial concentration of sulphuric acid and TOL.

\subsubsection{Experimental Characteristics}

(1) This reactive extraction-crystallization process is controlled by the circulating operation in fluidized bed crystallizer, which greatly simplifies the 
production technology and makes the operation convenient. It is conducive to cost reduction and resources saving.

(2) As shown in Figure 3, this novel crystallizer is similar to Oslo crystallizer. It must be noted that the bottom of the crystallizer is connected with pipes with different diameters. It is emphasized that a fundamental aim of this structure is to make the oil and aqueous phases fully mix and react in crystallizer. The extraction efficiency can be further promoted by this method.

(3) The buffer tank is mainly used in prolonging the residence time, improving the degree of the mixed solution phase-separation. Therefore, it can guarantee the experiments to go smoothly.

(4) In order to improve the flow pattern of mixed solution in SFBC and to remove the inclusion from solution in crystallizer, it also needs to be emphasized that the outlet size of crystallizer and phaseseparation tank is large, which is conducive to the timely flow of mixed solution at high circulation rate.

(5) Reducing the loss of extractant is the critical step in this process. The extractant has hydrophobic nonpolar group and hydrophilic polar group. The smaller the crystal size, the greater the surface energy, which makes the crystal easier to adsorb the oil phase. Therefore, in order to reduce the entrainment amount of extractant in the crystal, improve the product quality, and reduce the loss of extractant, it is necessary to obtain crystal with coarse and uniform distribution as far as possible. According to the characteristics of monolayer adsorption, the following equation is the ratio of the amount of extractant $(\omega)$ adsorbed to the crystals with particle sizes $d_{1}$ and $d_{2}$ $\left(d_{2}>d_{1}\right)$, respectively:

$$
\frac{\omega_{1}}{\omega_{2}}=\frac{6 \mathrm{~m} / \rho d_{1}}{6 \mathrm{~m} / \rho d_{2}}=\frac{d_{2}}{d_{1}} .
$$

It can be seen from equation (16) that the larger the obtained crystal size, the smaller the amount of adsorbed extractant, and the lower the loss of extractant, thereby reducing the production cost. Therefore, we need to increase the crystal size as much as possible, which can not only reduce the loss of extractant but also improve the quality of the final product.

In conclusion, we can see that oil and aqueous phases are well mixed in crystallizer, and there is no obvious selfseparation of the mixed solution during the entire process of the experiment. The phases of mixed solution can be spontaneously separated after entering the phase-separation tank so that oil and aqueous phases can be separated and recycled back to their phase slot, respectively. Mullin points out that adding an appropriate amount of pure sodium chloride as a seed crystal in crystallizer can promote crystal nucleation, thereby shortening the induction period of crystal nucleus and reducing the possibility of second nucleation [19].
To better understand the influence of operating conditions on CSD of crystals in this test, this paper uses the coefficient of variation (C.V.) to detect the quality of the crystal after the reactive extraction-crystallization experiment. In general, generated crystals vary not only in CSD but also in the coefficient of variation (C.V.). The C.V. is a statistical measure of the dispersion of data points in a data series around the crystal mean particle size [20]. The coefficient of variation represents the ratio of the standard deviation to the mean particle size, and it is a useful statistic for comparing the degree of variation from one data series to another, which reflects the uniformity of crystals [21]. The larger the values of C.V., the wider the CSD. On the contrary, the smaller the values of C.V., the narrower the CSD, and the more uniform the crystal size.

\section{Result and Discussion}

3.1. Preexperiment of Reactive Extraction-Crystallization. At different temperatures, the crystals with different sizes have different setting velocities in the supersaturated solution, which is caused by the drag force and gravity generated by the rising of the fluid on the crystals in SFBC. If the crystal rise velocity in the solution is equal to the settling velocity, it can be suspended in the solution. Generally speaking, the crystal rise velocity needs to be greater than the settling velocity to make the crystal suspend in the solution without causing "dead zone" in crystallizer. The different circulation rates of the crystallizer produce different rise velocities for the suspended crystals. Due to the impact of fluid in SFBC, the generated crystals will continue to be fluidized and suspended in the expanding zone of crystallizer. At this time, the size and number of crystals will be different under different heights of crystallizer. The value of settling velocity can be evaluated as a function relation of crystal volume concentrations from the correlation reported by Richardson and Zaki [22]:

$$
v_{g}=v_{0}\left(1-C_{V}\right)^{n-1},
$$

where $v_{g}$ is the hindered settling velocity, $v_{0}$ is the free settling velocity, $C_{V}$ is the volume concentration of crystals, and $n-1$ represents the experimental fitting index. The experimental fitting index is estimated as the function of the Reynolds number by Garside and Al-Dibouni [23]:

$$
n=\frac{5.1+0.27 \mathrm{Re}^{0.5}}{1.0+0.1 \mathrm{Re}^{0.9}} .
$$

Therefore, we need to investigate the settling velocity of crystals with different sizes at different temperatures, so as to select the appropriate circulation rate.

It can be seen from Figure 4 that the setting velocity of sodium chloride crystals decreases with the increase in temperature. Lower viscosity of the solution was associated with higher temperature. Meanwhile, the setting velocity of crystals is proportional to the viscosity of the solution. It can be seen that if the temperature is too high during the experiment, the setting velocity of crystals in solution will decrease. Once the values of setting velocity drop to the 


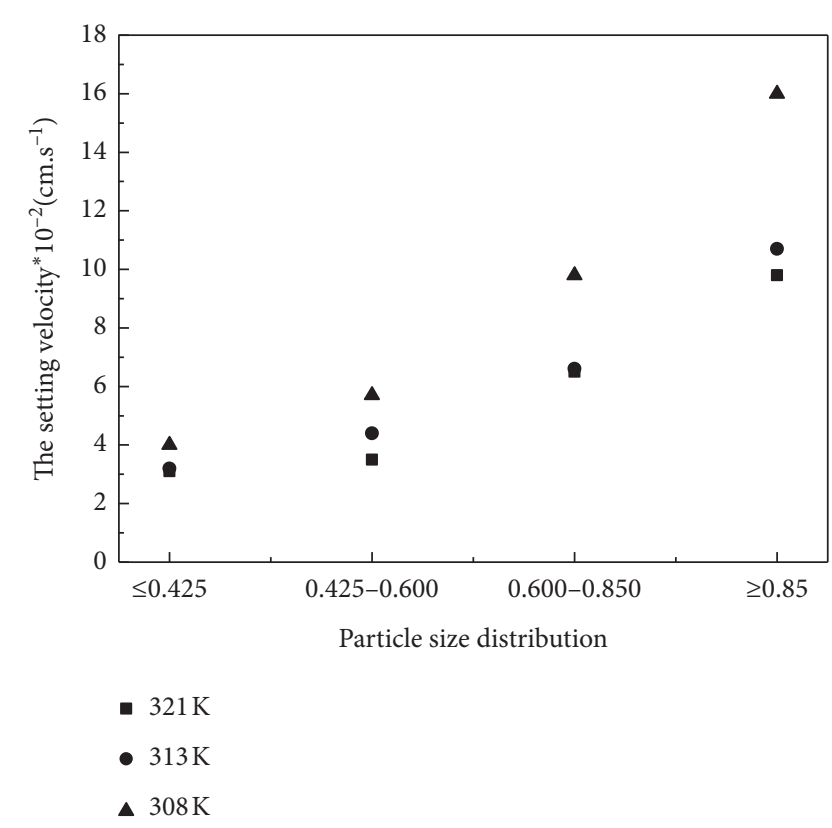

Figure 4: Setting velocity of sodium chloride at different temperatures.

point where it is unable to balance with the fluidization velocity of crystals, the generated sodium chloride crystals will be easily carried out by the fluid, resulting in the loss of product quality. Therefore, the setting velocity of crystal should not be too small. On the contrary, as the crystal grows, the setting velocity of crystals increases. Once it is greater than the minimum fluidization rate, the fluidized state of crystallizer bed layer is likely to change from the fluidized bed back to the fixed bed again, which will eventually affect the crystal quality. It is necessary to control the circulation rate strictly to balance the crystal settling velocity in SFBC. Therefore, the reasonable control of circulation rate is a key step in the experiment.

\subsection{Experimental Studies on the Extraction Process}

3.2.1. Effect of Technological Parameters on Extraction Rate. It can be seen from Figure 5 that when the circulation flow rate is less than $1.0362 \mathrm{~m} / \mathrm{s}$, the extraction rate increases with the increase in circulation flow rate; when the flow rate is greater than $1.0362 \mathrm{~m} / \mathrm{s}$, the extraction rate decreases, thus affecting the extraction efficiency. When the contact area of oil and aqueous phases is constant, the extraction rate is directly proportional to the circulation rate. However, when the circulation flow rate is greater than a certain value, the contact reaction time of the two phases decreases, which affects the extraction efficiency. Therefore, the extraction of sulphuric acid by TOL is likely to be a diffusion-controlled process. In order to ensure that the two phases of solution can fully contact with each other and do not affect the extraction efficiency, we select $1.0362 \mathrm{~m} / \mathrm{s}$ as the optimal circulation flow rate. Figure 6(a) shows that the extraction rate increases as the concentration of sulphuric acid increases. Figure 6(b) indicates the effect of concentration of

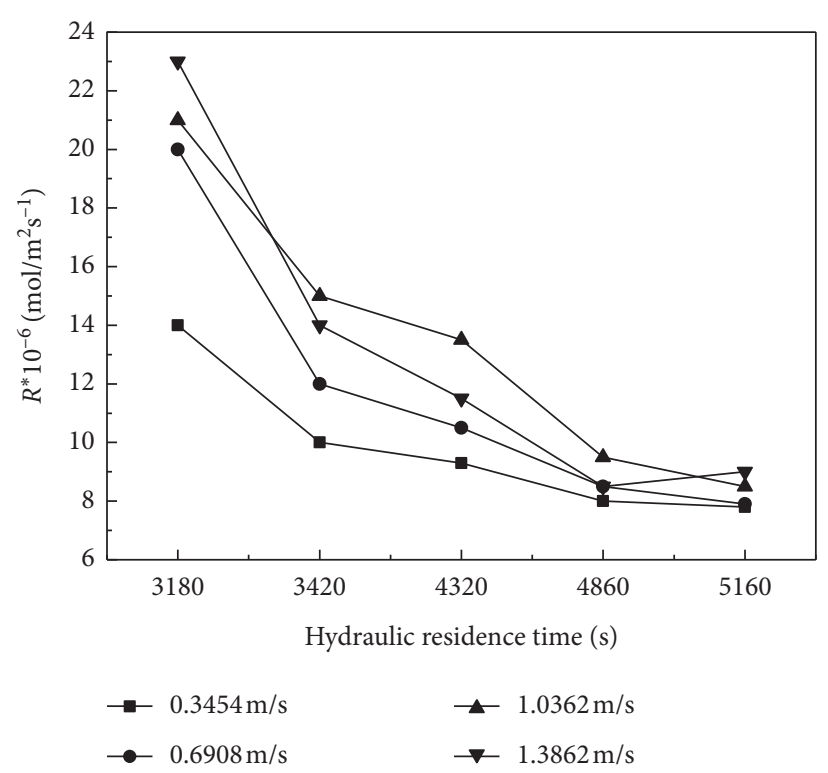

FIGURE 5: Effect of circulation flow rate on extraction rate.

TOL revealing that the extraction rate first increases and then decreases with the increasing concentration. The extraction rate is the highest when the concentration is 0.8 moL. $\mathrm{L}^{-1}$. When the concentration is beyond $0.8 \mathrm{moL} . \mathrm{L}^{-1}$, the viscosity of the oil phase increases progressively because the impact of diluents becomes progressively weaker, leading to the deceleration of the reaction extent.

\subsubsection{Effect of Different Temperatures on Extraction Rate} Constants. The temperature is the important factor in the research of extraction kinetics. By studying the effect of temperature on extraction rate, we can deeply understand the extractive reaction system and obtain the thermodynamic data such as the activation energy of extractive reaction. If the activation energy of the extractive reaction is greater than $40 \mathrm{KJ} / \mathrm{mol}$, the extraction process is controlled by a chemical reaction. When the activation energy is less than $20 \mathrm{KJ} \mathrm{mol}^{-1}$, the extraction process is controlled by diffusion reaction. It can be seen from Figure 7 that the extraction rate constant increases with the increase in temperature, indicating that high temperature is conducive to the extractive reaction and accelerates the mass transfer rate. According to Arrhenius equation $k=k_{0} \exp (-E / \mathrm{RT})$, the activation energy of extraction is $24.9 \mathrm{KJ} \mathrm{mol}^{-1}$, which proves that the system is controlled by diffusion and chemical reaction. On the contrary, high temperature may lead to the failure of the extractant, so the selection of temperature should be based on the experimental results of crystal size distribution.

3.2.3. Comparison of Nucleation Rate. As shown in Table 2, the application of nonlinear regression analysis in establishing extraction kinetic model that the extraction kinetic model demonstrates describes the extraction rate data well. The correspondence among experimental and extraction kinetic rate is shown in Figure 8. As shown 


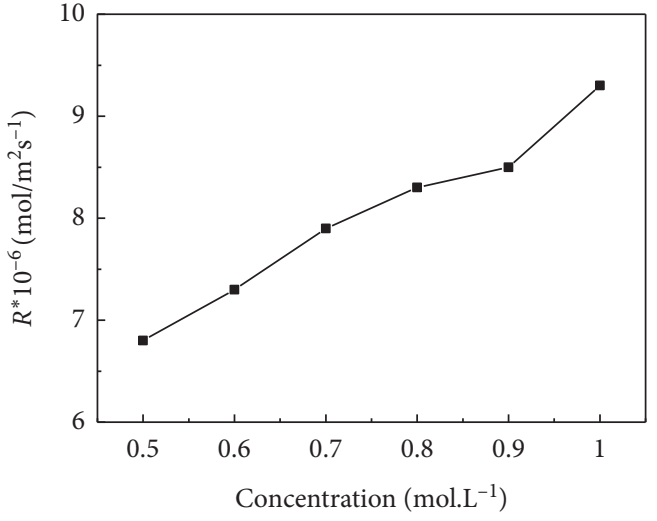

(a)

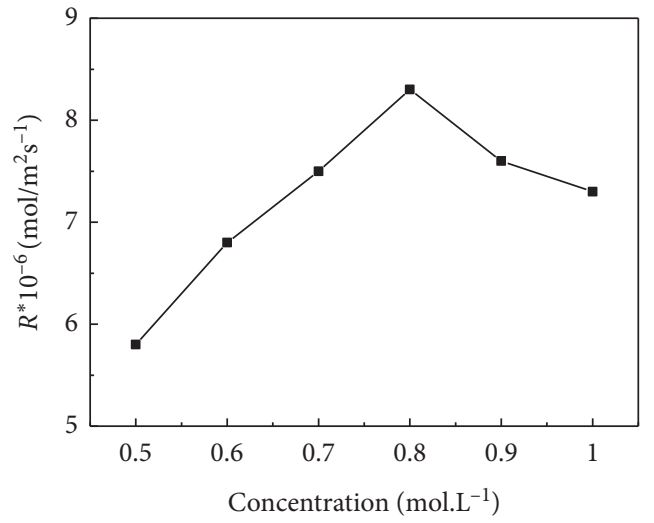

(b)

FIGURE 6: Effect of solution concentration on extraction rate: (a) sulphuric acid; (b) TOL.

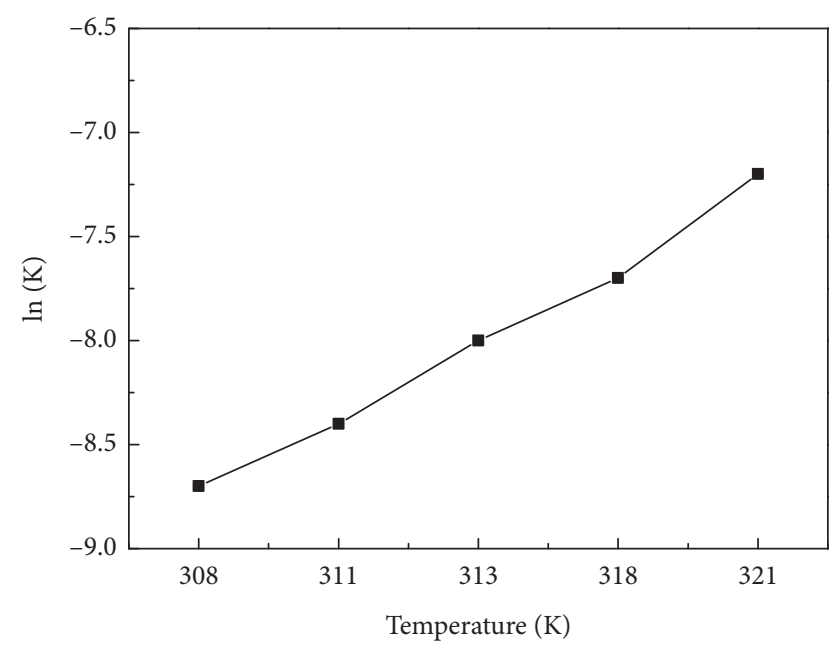

Figure 7: Effect of different temperatures on extraction rate constants.

TABLe 2: Parameters of the extraction rate for equation (15).

\begin{tabular}{lccc}
\hline Circulation flow rate & $K$ & $K^{+}$ & $R^{2}$ \\
\hline $1.0362 \mathrm{~m} / \mathrm{s}$ & $4.376 * 10^{-5}$ & $3.781 * 10^{-6}$ & 0.9875 \\
\hline
\end{tabular}

previously, the extraction kinetic model suggests that the extraction rate is proportional to the concentration of sulphuric acid. As can be seen, compared with the kinetic model, the extraction rate of sulphuric acid predicted by equation (15) is demonstrated to correlate more closely to the experimental results, deviating by less than $15 \%$. The $\mathrm{K}$ value is much higher than the $\mathrm{K}^{+}$value, which indicates that the mass transfer rate of sulphuric acid in the aqueous phase is much higher than that at the phase interface.

3.3. Experimental Studies on the Crystal Size Distribution of Sodium Chloride. Since the entire crystallization process is controlled by the continuous operation, the oil and aqueous phases are continuously flowing, mixing, and reacting in

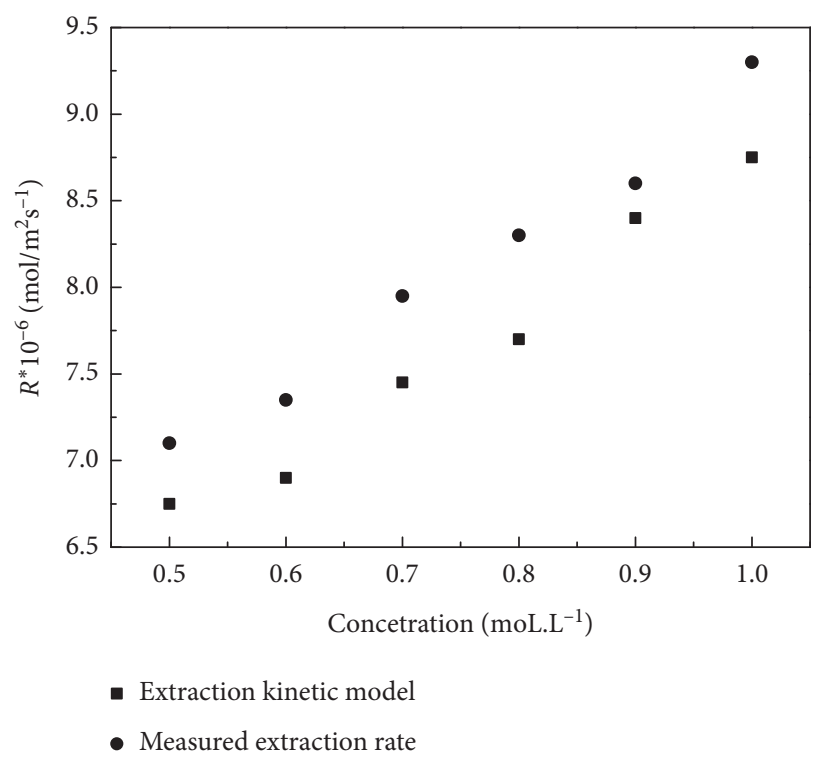

FIGURE 8: Comparison of the predicted and measured extraction rate.

crystallizer. If the solution supersaturation produced by the crystallization process is not evenly absorbed by the suspended crystals, it will cause excessive local supersaturation and produce fine grains in crystallizer. Obviously, these are not the results we expect. In addition, a partial channeling in the bed will occur if the crystals are not fully fluidized in the fluidized bed, which will cause some particles to accumulate at the bottom of the crystallizer. It not only affects the quality of the crystal products but also is not conducive to the crystallization operation. Furthermore, the crystal block at the bottom of the crystallizer needs to be removed frequently, which actually increases the production cost. Therefore, it is necessary to explore the optimal conditions to facilitate the crystallization operation. The effects on the reactive extraction-crystallization process are quantified by single-factor experiments in order to determine the optimal conditions for the coupled process. 
3.3.1. Effect of Velocity Ratios of Oil and Aqueous Phases. The velocity ratio between organic phase and aqueous phase has an important effect on the reactive extraction-crystallization process. The experiments are performed with five velocity ratios, respectively, and the results are shown in Figure 9. The results indicate that there are some differences among the size distributions when the velocity ratio is $9.5: 8$ and the crystal main size is about $0.425-0.600 \mathrm{~mm}$. This may be due to the fact that the beds of solids fluidize poorly with bumping and spouting in the SFBC under this velocity ratio of oil and aqueous phases, resulting in the insufficient growth of crystal. In addition, according to the results, we concluded that, under the same experimental conditions, as the velocity ratio decreases, the crystals become larger. Obviously, the crystal size distribution of the latter four groups is relatively uniform. In order to indicate the difference crystal size distribution visually, it is necessary to focus on calculating the coefficient of variation (C.V.) of crystals in different phase ratios. The C.V. of crystals is defined by the following equation [24]:

$$
C . V .=\frac{50\left(\mathrm{PD}_{84 \%}-\mathrm{PD}_{16 \%}\right)}{\mathrm{PD}_{50 \%}},
$$

where $\mathrm{PD}_{m \%}$ is the aperture size with cumulative mass fraction of $\mathrm{m} \%$.

Table 3 list the C.V. of the product under different velocity ratios, revealing that, with the velocity ratio being 9.5 : 8.5 , the C.V. of crystals is the lowest, the crystal quality is the best, and the crystal size distribution is the most uniform. However, it should be noted that the velocity ratio should not be too large because the large velocity ratio will lead to poor mixing effect of oil and aqueous phases in the extraction zone of the crystallizer. If the acid in solution cannot be extracted in time and increases the acidity of the mother liquor, this will not only prolong the extraction time, and it will also lead to the generation of fine grains dissolved in the mother liquor, which will affect the reactive extractioncrystallization process. Consequently, we select $9.5: 8.5$ as the optimal velocity ratio.

3.3.2. Effect of Temperature. In order to investigate the influence of temperature on CSD in the reactive extractioncrystallization system, experiment is performed at temperature values from $308 \mathrm{~K}$ to $321 \mathrm{~K}$, while all other parameters are kept constant; the results are shown in Figure 10. The pronounced change in crystal size with temperature has been confirmed by determining the corresponding CSD. It can be seen that there are no obvious differences among the size distributions, and the mass fraction of main crystal size increases initially and then decreases off with temperature increase. The mass fraction of main crystal size varies from $53 \%$ at $311 \mathrm{~K}$ to $56 \% \mathrm{~m}$ at $313 \mathrm{~K}$ and $52 \%$ at $321 \mathrm{~K}$. Overall, a moderate increase in temperature is conducive to crystallization. The extraction process is a spontaneous endothermic reaction. Accordingly, adequate increase in bed temperature is helpful for the extraction. However, when the temperature is higher than $313 \mathrm{~K}$, the effect of temperature on the nucleation and growth of the crystal is not obvious,

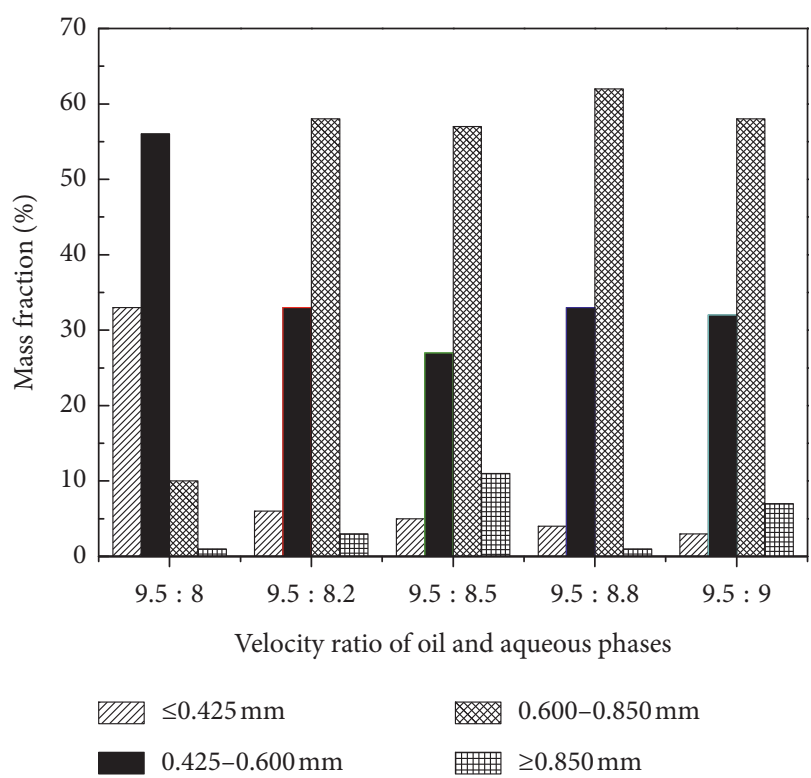

Figure 9: Effect of different velocity ratios of oil and aqueous phases on crystal size distribution.

TABLE 3: C.V. of the crystals with different velocity ratios of oil and aqueous phases.

\begin{tabular}{ll}
\hline Velocity ratio & C.V. \\
\hline $9.5: 8.2$ & 40.12 \\
$9.5: 8.5$ & 32.56 \\
$9.5: 8.8$ & 34.55 \\
$9.5: 9$ & 37.43 \\
\hline
\end{tabular}

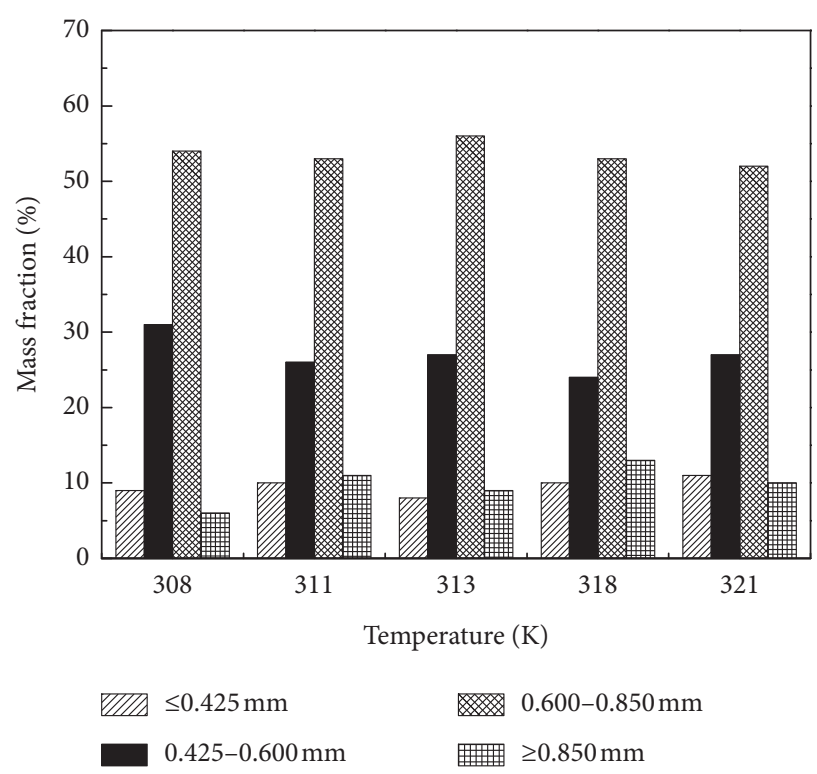

Figure 10: Effect of different temperatures on crystal size distribution. 
which can also be reflected from the C.V. of crystals (Table 4). The kinetic coefficient of sodium chloride crystallization is not sensitive to temperature, and thus, it is not possible to control the main crystal size by means of adjusting the temperature. Meanwhile, a too high temperature is unfavorable for the reactive extraction-crystallization process and results in high energy consumption. From this perspective, the temperature of $313 \mathrm{~K}$ is selected as the most appropriate optimized condition.

3.3.3. Effect of Hydraulic Residence Time. The crystal size distributions of sodium chloride generated at different hydraulic residence times are displayed in Figure 11. The reaction equilibrium is achieved after a long contact time due to the complicated liquid-liquid-solid phase system. It is pretty obvious that the mass fraction of the main crystal size with a longer residence time is larger than that of the crystal with a shorter residence time. Meanwhile, the mass fraction of the small crystal gradually decreases with the increase in the residence time under the residence time less than $4320 \mathrm{~s}$. This indicates that the growth of large crystals is fast while that of small crystals is relatively slow during this growth process. This is probably because the setting velocity of largesize crystals is high, and the solution contacting at the bottom of the crystallizer has a high supersaturation. These factors promote the growth of large crystals. In the meantime, the main crystal size can be effectively increased by extending residence time. However, it should be noted that when the crystal size increases to some extent, the generated crystals need to be taken out in time to avoid clogging of crystallizer, which can ensure the stability of the crystal size distribution in the fluidized bed. Additionally, the coefficients of variation in crystal exceed $35 \%$ except $4320 \mathrm{~s}$ in Table 5. Considering the abovementioned factors comprehensively, the hydraulic residence times of $4320 \mathrm{~s}$ is selected as the optimal experimental condition.

3.3.4. Effect of Feed Velocity. Furthermore, the effect of feeding velocities on the CSD is also investigated in this study. The sulphuric acid is continuously dropped into the sodium sulfate solution. The size distributions of the generated sodium chloride are shown in Figure 12. As shown in Figure 12, the mass fraction of the crystal main size with a higher feeding velocity is greater than that of the product with a slower feeding velocity. This is because the crystal growth rate increases with the increase in feeding velocity. However, the reaction time between the oil and aqueous phases is reduced due to too fast feeding when the feeding rate is greater than $8 \mathrm{~mL} \cdot \mathrm{min}^{-1}$, resulting in the decrease in the final crystal size. Meanwhile, the increase in feeding velocity will also increase the extraction reaction rate between the oil and aqueous phases, resulting in excessive local supersaturation in SFBC. This leads to an increase in the amount of fine crystals and a decrease in the mass fraction of crystal main size. The value of crystal C.V. is the lowest when the feed velocity is $8 \mathrm{~mL} \cdot \mathrm{min}^{-1}$ in Table 6 . Consequently, we select $8 \mathrm{~mL} \cdot \mathrm{min}^{-1}$ as the optimal feeding velocity.
TABLE 4: C.V. of the product with different temperatures.

\begin{tabular}{ll}
\hline Temperature $(\mathrm{K})$ & C.V. \\
\hline 308 & 33.75 \\
311 & 32.98 \\
313 & 32.15 \\
318 & 35.22 \\
321 & 36.76 \\
\hline
\end{tabular}
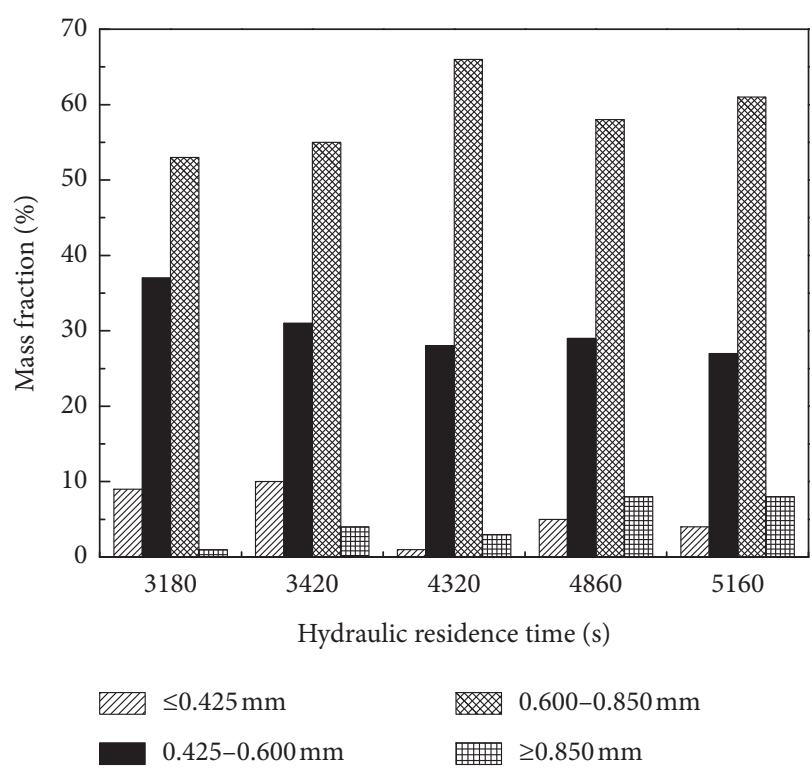

FIGURE 11: Effect of different hydraulic residence times on crystal size distribution.

TABle 5: C.V. of the product with different hydraulic residence times.

\begin{tabular}{lc}
\hline Hydraulic residence times (s) & C.V. \\
\hline 3180 & 38.67 \\
3420 & 36.11 \\
4320 & 33.95 \\
4860 & 35.43 \\
5169 & 35.23 \\
\hline
\end{tabular}

Therefore, the optimal conditions in the coupled reactive extraction-crystallization process, as obtained by the singlefactor experiments, are as follows: velocity ratio of oil and aqueous phases $9.5: 8.3$, temperature $313 \mathrm{~K}$, hydraulic reaction time $4320 \mathrm{~s}$, and feed velocity $8 \mathrm{~mL} \cdot \mathrm{min}^{-1}$.

\subsection{Comparative Experiment}

3.4.1. Experimental Procedure. In the last part of this paper, we do a contrast test between different crystallization patterns. As shown in Figure 1, the reaction extraction in a stirred crystallizer is conducted in batch-mode crystallization. The supersaturated solution is prepared by dissolving a defined amount of sodium chloride in deionized water. The solution is filtered before crystallization to guarantee that the 

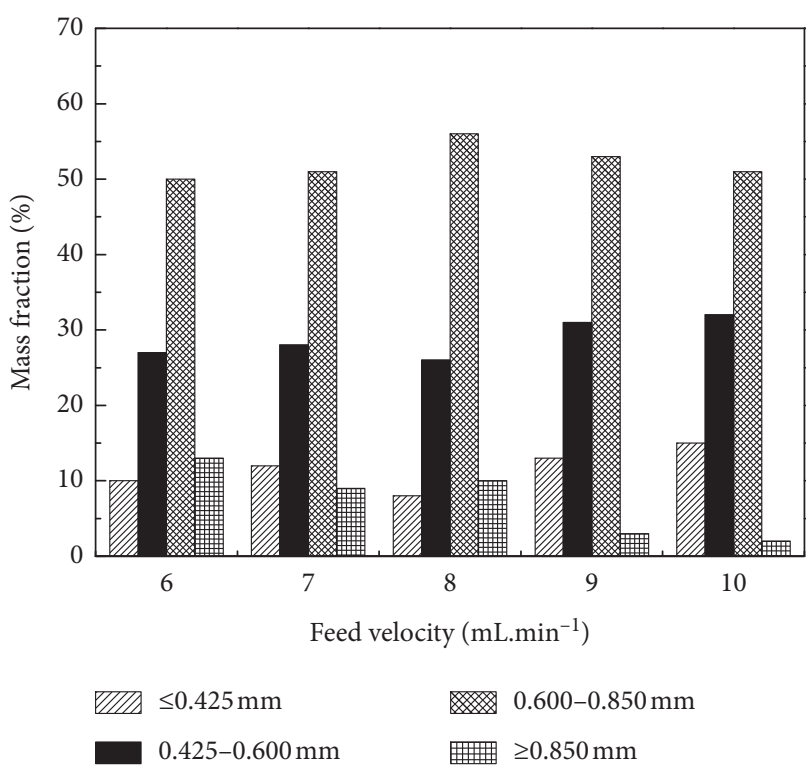

Figure 12: Effect of different feed velocities on crystal size distribution.

TABLE 6: C.V. of the product with different feed velocities.

\begin{tabular}{lc}
\hline Feed velocity $\left(\mathrm{mL} \cdot \mathrm{min}^{-1}\right)$ & C.V. \\
\hline 6 & 37.37 \\
7 & 35.65 \\
8 & 33.22 \\
9 & 34.43 \\
10 & 36.97 \\
\hline
\end{tabular}

solution is clear and free of impurities. Turn on the peristaltic pump to transport the oil and aqueous phases into the crystallizer after adjusting the speed of the impeller. Subsequently, a part of the solution is drawn at regular intervals for $\mathrm{pH}$ measurement after the two phases of the solution are fully mixed in a stirred crystallizer and thorough stirring throughout the $0.5 \mathrm{~h}$ experiment.

3.4.2. Effect of Stirring Rate. In stirring extraction, the stirring rate has a great influence on particle size. When the other experimental conditions are constant, the mass fraction of crystal main size shown in Figure 13 decreases with increasing stirring rate, which may be due to shear stress. On the contrary, the results show that the mass fraction of the crystal main size is about less than $40 \%$, which is less than that of the fluidized crystallization under the same experimental conditions. The higher the stirring rate, the larger the shear stress, resulting in more fine grains in a stirred crystallizer. Although the high stirring rate is beneficial to the crystal growth process, there are still some crystals that only move at the bottom of the crystallizer without growth. On the contrary, the probability of collision between crystal and impeller is increased, and secondary nucleation is more likely to occur in a stirred crystallizer.
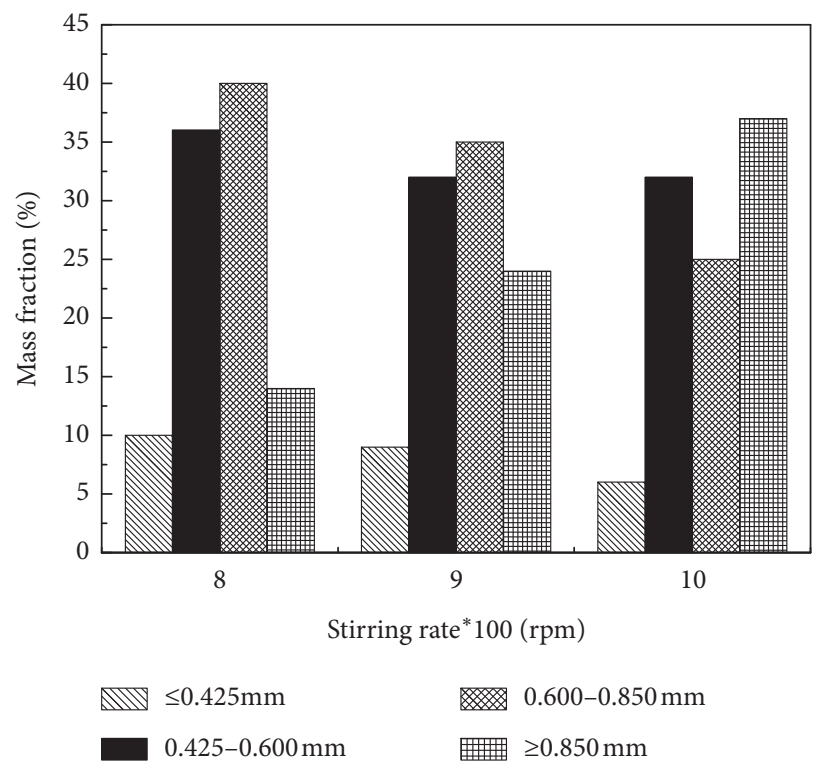

FIgURE 13: Effect of different phase ratios on crystal size distribution.

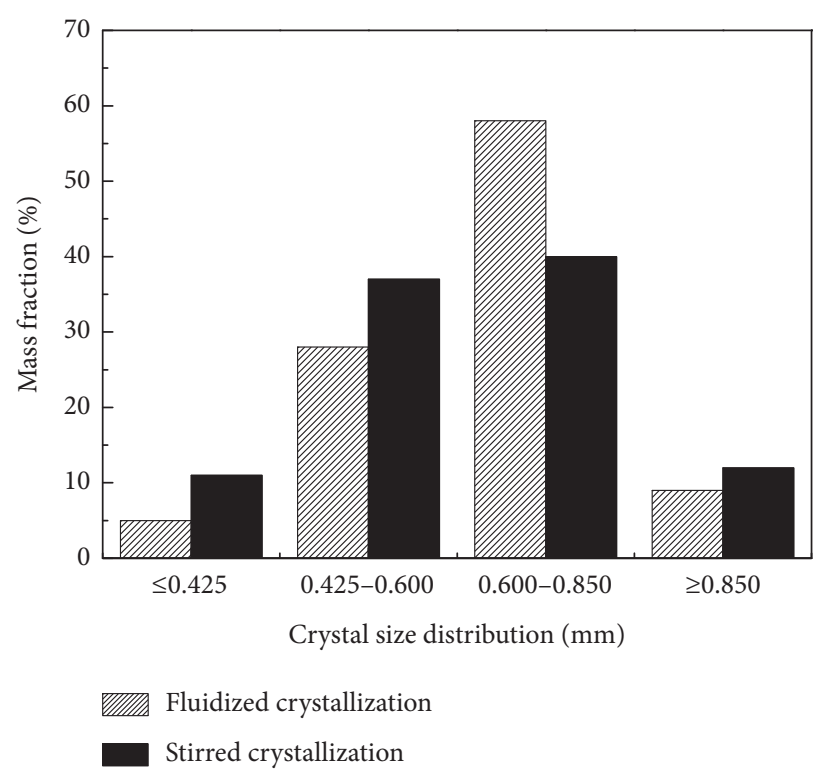

FIgURE 14: Effect of different phase ratios on crystal size distribution.

3.4.3. Effect of Phase Ratio. Figure 14 displays the mass fraction of the crystal main size produced by fluidized crystallization is obviously larger than that of the stirred crystallization, while the mass fraction of the small crystal is smaller than that of the stirred crystallization under the same phase ratio. In a stirred crystallizer, the shear stress between crystals and impeller is large, which would cause particles to collide fiercely. In addition, the induction period of smalldiameter seeds is longer, so crystal growth consumes more 
supersaturation, thereby inhibiting the generation of crystal nuclei.

\section{Conclusion}

In this research, the multiphase reactive extraction-crystallization process is investigated systematically in order to offer a novel and useful perspective for the crystallization of sodium chloride in a SFBC. The optimal conditions in the coupled reactive extraction-crystallization process, as obtained by the single-factor experiments, are as follows: circulation flow rate $1.0362 \mathrm{~m} / \mathrm{s}$, velocity ratio of oil and aqueous phases $9.5: 8.5$, temperature $313 \mathrm{~K}$, hydraulic reaction time $4320 \mathrm{~s}$, and feed velocity $8 \mathrm{~mL} \cdot \mathrm{min}^{-1}$. In the experiment, we should pay attention to the fact that the residence time of the mixed solution is too short due to the excessive circulation rate in crystallizer, which leads to the solution not being separated in time in the phase-separation tank, resulting in poor extraction effect. Meanwhile, this work proves that the reactive extraction system is controlled by diffusion and chemical reaction. The proposed extraction kinetic model about extraction rates is developed and validated against data from the SFBC. Analysis of the extraction kinetic model and comparison with experiments reveals that the extraction kinetic model results are in well agreement with the experiment. In a final step, the values of CSD are taken as the index and compared with the experimental data in a stirred crystallizer. Operating the SFBC in the continuous mode increases the mass fraction of crystal main size in the solution. The experimental results provide valuable information for future crystallization process operations.

\section{Data Availability}

All data generated or analyzed during this study are included in this published article. The data included in this study are available upon request from the corresponding author.

\section{Conflicts of Interest}

The authors declare that they have no conflicts of interest.

\section{Acknowledgments}

This work was supported by the Foundation for High-Level Talents in Higher Education of Sichuan University of Science and Engineering (no. 2017RCL68), Sichuan Science and Technology Program (no. 2020YFG0163), Undergraduate Training Program for Innovation and Entrepreneurship (no. S201910622083), and the Opening Project of Key Laboratories of Fine Chemicals and Surfactants in Sichuan Provincial Universities (no. 2019JXY01).

\section{References}

[1] Y. Li, X. Song, G. Chen, Z. Sun, Y. Xu, and J. Yu, "Preparation of calcium carbonate and hydrogen chloride from distiller waste based on reactive extraction-crystallization process," Chemical Engineering Journal, vol. 278, pp. 55-61, 2015.
[2] M. Weber, M. J. Jones, and J. Ulrich, "Optimisation of isolation and purification of the jack bean enzyme urease by extraction and subsequent crystallization," Food and Bioproducts Processing, vol. 86, no. 1, pp. 43-52, 2008.

[3] A. T. Mbambo, O. Stanley, P. S. Mdluli, and M. M. Lawrence, "Development of a gold nanomaterial enabled colorimetric sensor method for the analysis of sodium chloride in seawater," Environmental Nanotechnology Monitoring \& Managemen, vol. 12, 2019.

[4] A. M. Gibson, N. Bratchell, and T. A. Roberts, "The effect of sodium chloride and temperature on the rate and extent of growth of Clostridium botulinum type A in pasteurized pork slurry," Journal of Applied Bacteriology, vol. 62, no. 6, pp. 479-490, 2010.

[5] T. Trautmann, B. Corinna, F. Andre, V. Deirdre, R. Konrad, and B. Michael, "The impact of oral sodium chloride supplementation on thrive and the intestinal microbiome in neonates with small bowel ostomies: a prospective study," Frontiers in Immunology, vol. 11, 2020.

[6] M. Inaba, H. Shohei, L. Henan, K. Mikoto, N. Michihiko, and S. Junya, "Dielectrophoretic properties of submicron diamond particles in sodium chloride aqueous solution," Japanese Journal of Applied Physics, vol. 59, no. 4, 2020.

[7] J. Halambek, I. Cindrić, and A. Ninčević Grassino, "Evaluation of pectin isolated from tomato peel waste as natural tin corrosion inhibitor in sodium chloride/acetic acid solution," Carbohydrate Polymers, vol. 234, p. 115940, 2020.

[8] Y. Li, X. Song, G. Chen, and Z. Sun, "Preparation of calcium carbonate and hydrogen chloride from distiller waste based on reactive extraction-crystallization process," Chemical Engineering Journal, vol. 278, pp. 55-61, 2015.

[9] M. V. S. R. Ravi Kanth, S. Pushpavanam, S. Narasimhan, and B. Narasimha Murty, "A thermodynamic model for reactive extraction of macro amounts of zirconium and hafnium with TBP," Separation and Purification Technology, vol. 240, p. 116491, 2020.

[10] A. Coenen, "Process for preparing sodium bicarbonate and hydrogen chloride," 1982.

[11] D. Zheng, T.-H. Huang, J. Yan, and Y. Hu, "Coupling of abrasion attrition theory with mechanical characteristics for particle attrition in a fluidized bed reactor," Asia-Pacific Journal of Chemical Engineering, 2020.

[12] D. Zheng, J. Li, Y. Jin, D. Zou, and X. Zhu, "Coupling of CFD with PBM for growth behavior of potassium sulphate in spray fluidized-bed crystallizer," Powder Technology, vol. 314, pp. 427-441, 2017.

[13] S. Biswas, A. K. Biswas, and B. De, "Influence of sodium chloride on growth and metabolic reprogramming in nonprimed and haloprimed seedlings of blackgram (Vigna mungo L.)," Protoplasma, vol. 257, pp. 1-25, 2020.

[14] P. Pronk and C. A. Infante Ferreira, "Witkamp, Mitigation of ice crystallization fouling in stationary and circulating liquid-solid fluidized bed heat exchangers," International Journal of Heat and Mass Transfer, vol. 53, no. 1-3, pp. 403-411, 2010.

[15] D. Zheng, W. Zou, C. Peng, Y. Fu, J. Yan, and F. Zhang, "CFD-PBM coupled simulation of liquid-liquid dispersions in spray fluidized bed extractor: comparison of three numerical methods," International Journal of Chemical Engineering, vol. 2019, pp. 1-13, Article ID 4836213, 2019.

[16] D. Zheng, W. Zou, J. Yan, and C. Peng, "A comparative study on the nucleation, growth, and agglomeration kinetics of potassium dihydrogen phosphate crystal in circulating 
fluidized-bed crystallizer," Asia-Pacific Journal of Chemical Engineering, vol. 14, no. 6, 2019.

[17] A. P. T. Rocha, H. M. Lisboa, O. L. S. Alsina, and O. S. Silva, "Coating process of Phyllanthus niruri Linn granules using spouted bed," Powder Technology, vol. 336, pp. 85-91, 2018.

[18] M. D. G. de Luna, D. P. M. Rance, L. M. Bellotindos, and M.-C. Lu, "A statistical experimental design to remove sulfate by crystallization in a fluidized-bed reactor," Sustainable Environment Research, vol. 27, no. 3, pp. 117-124, 2017.

[19] J. Kluge, L. Joss, S. Viereck, and M. Mazzotti, "Emulsion crystallization of phenanthrene by supercritical fluid extraction of emulsions," Chemical Engineering Science, vol. 77, pp. 249-258, 2012.

[20] M. J. Bos, A. M. Alzate Sanchez, R. Bancone et al., "Influence of anesthesia and clinical variables on the firing rate, coefficient of variation and multi-unit activity of the subthalamic nucleus in patients with Parkinson's disease," Journal of Clinical Medicine, vol. 9, no. 4, p. 1229, 2020.

[21] D. Zheng, W. Zou, J. Yan et al., "Coupling of contact nucleation kinetics with breakage model for crystallization of sodium chloride crystal in fluidized bed crystallizer," Journal of Chemistry, vol. 2019, pp. 1-11, 2019.

[22] J. F. Richardson and W. N. Zaki, "Sedimentation and fluidization: Part I," Transactions of the Institution of Chemical Engineers, vol. 32, pp. 35-47, 1954.

[23] J. Garside and M. R. Al-Dibouni, "Velocity-voidage relationships for fluidization and sedimentation in solid-liquid systems," Industrial \& Engineering Chemistry Process Design and Development, vol. 16, no. 2, pp. 206-214, 1977.

[24] M. S. Arulampalam, S. Maskell, N. Gordon, and T. Clapp, “A tutorial on particle filters for online nonlinear/non-Gaussian bayesian tracking," IEEE Transactions on Signal Processing, vol. 50, no. 2, pp. 174-188, 2002. 